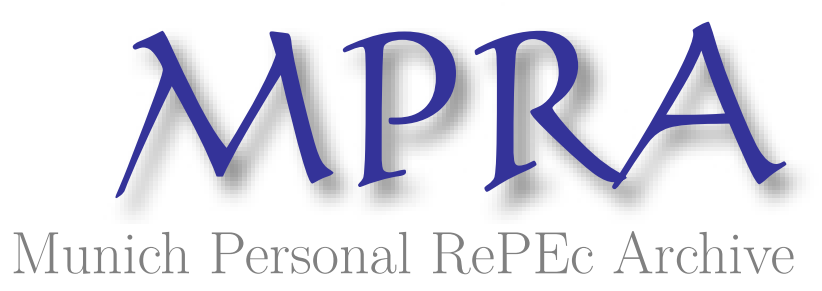

Disentangling non-linearities in the longand short-run price relationships: An application to the U.S. hog/Pork supply chain

Gervais, Jean-Philippe

Laval University

10 April 2007

Online at https://mpra.ub.uni-muenchen.de/7743/

MPRA Paper No. 7743, posted 13 Mar 2008 18:56 UTC 


\title{
Disentangling Non-linearities in the Long- and Short-run Price Relationships: An Application to the U.S. Hog/Pork Supply Chain ${ }^{1}$
}

\author{
Jean-Philippe Gervais \\ Canada Research Chair in Agri-industries and International Trade \\ CRÉA and Department of Agricultural Economics \\ and Consumer Studies, Laval University
}

This version, January 2008

Previous version, April 2007

\begin{abstract}
Increased concentration at the retail, food processing and farm input manufacturing levels has brought increased attention to patterns in retail-to-farm price spreads. Most studies documenting asymmetric price transmission focus on non-linear error correction processes, as opposed to the current study which analyzes potential non-linearities in the long-run relationship between the farm and retail prices. The null hypothesis of non-linearity in the long-run relationship between farm and retail prices in the U.S. hog/pork supply chain is rejected in favor of a Smooth Transition Cointegration (STC) framework. The STC framework predicts downward price stickiness in retail prices. The predicted residuals of the non-linear model are used to investigate whether it is possible to disentangle non-linearity in the long-run price relationship from non-linearity in the adjustment towards the long-run equilibrium. The results underline the importance of testing for linearity in the long-run price relationship before modeling non-linearity in short-run dynamics.
\end{abstract}

Keywords: Smooth transition cointegration; farm to retail price transmission; linear cointegration.

J.E.L. Classification: C22; Q11

1 Contact information is 2425 Rue de l'agriculture, Office 4415, Department of Agricultural Economics, Laval University, Quebec City, QC, Canada, G1V 0A6. Phone: (418) 656-2131. Fax: (418) 656-7821. E-mail: Jean-Philippe.Gervais@eac.ulaval.ca. I am thankful for the comments and suggestions of three anonymous reviewers. I would also like to thank Barry Goodwin for providing useful comments on an earlier draft of this manuscript. The usual caveat about remaining errors applies. Part of this work was carried out when I was on sabbatical leave at North Carolina State University and I am particularly thankful for the hospitality of the Department of Agricultural and Resource Economics. 


\section{Disentangling Non-Linearities in the Long- and Short-run Price Relationships: An Application to the U.S. Hog/Pork Supply Chain}

\section{Introduction}

There exists a widespread belief in the farm sector that price transmission in agri-food supply chains is asymmetric. Increases in farm prices are believed to be matched faster at the retail level whereas negative shocks at the farm level take more time to be passed on to consumers. These concerns have recently been validated to some extent in the literature (see for example Azzam, 1999; Abdulai, 2002; Serra and Goodwin, 2003). Important shocks to agri-food supply chains (e.g., the 2003 Canadian mad cow incident or the 1998 North American hog crisis) usually amplify the perceived problems associated with price transmission. Concentration in downstream markets and input manufacturing industries are often pointed out (perhaps wrongly) to explain why in certain instances, decreases in upstream prices are not accompanied by proportional decreases in downstream prices. There are however other possible explanations for Asymmetric Price Transmission (APT) such as inventory management, menu costs of changing prices, and other adjustment costs (Meyer and von Cramon-Taubadel, 2004).

The purpose of the paper is to propose a procedure to investigate potential asymmetries in price transmission. It focuses on the relationship between farm and retail prices in the U.S. hog/pork industry. U.S. hog production has considerably evolved since the early 1980s as vertically coordinated operations replaced small and independently owned farm which used to sell their output to unspecialized packing/processing plants (Haley 2004). The increased reliance on contractual arrangements between producers and packers combined with increasing concentration in packing activities (MacDonald and Ollinger, 2000) led to concerns that market conditions are more conducive to the exercise of market power by retailers and processors. 
Frey and Manera (2007) provide an extensive review of price transmission and define the concept of APT from an empirical perspective as measuring whether output prices respond symmetrically to variations in input prices. APT in magnitude generally refers to the response in the output price made conditional on the direction of the change in the input price. APT in speed refers to the pace of the response in the output price made conditional on the direction of the change in the input price.

There exists a growing literature on the issue of price transmission in agri-food supply chains. The common feature in most of the empirical studies is that asymmetry is related to the speed of transmission. Price shocks in upstream and downstream markets eventually error correct and prices eventually drift back to a stable long-run equilibrium. However, a positive shock will entail a different adjustment path towards the equilibrium than a negative one under APT in speed. For example, Goodwin and Holt (1999) and Serra and Goodwin (2003) used threshold cointegration techniques to study price transmission in the U.S. beef and Spanish dairy industries respectively. They found that farm and retail prices are tied by a linear long-run relationship and that the adjustment path towards the equilibrium is asymmetric. Chavas and Mehta (2004) investigated asymmetric short-run price dynamics for dairy products in a linear cointegration model.

There have been however some attempts to capture potential asymmetries in the magnitude of price transmission. Miller and Hayenga (2001) investigated price transmission in the frequency domain by dividing their sample into low and high frequency price cycles. In a second stage, the authors uncovered asymmetric price transmission in the time domain. They did not address however the stochastic properties of the data and the possibility prices were cointegrated. Lass (2005) used linear methods applied to non-linear transformations of integrated variables. While conventional inference rejected the null hypothesis of symmetric price transmission in speed and magnitude, it is 
not known if his statistical results are robust when the appropriate limiting distribution theory is applied (Park and Phillips, 2001).

The objective of the paper is to provide a first investigation of price transmission focusing on potential non-linearities in both the long- and short-run price relationships. The presence of non-linearity is a necessary condition for the existence of APT, but is not sufficient as will be illustrated in the next section. In the context of cointegration, a nonlinear long-run relationship between prices can lead to APT in magnitude, while APT in speed can be captured by a non-linear adjustment process towards the long-run equilibrium. We thus propose a framework to separate out non-linearity in the long-run relationship from non-linearity in the adjustment path towards the long-run equilibrium.

\section{A cursory review of asymmetric price transmission}

Most of the early empirical specifications to uncover asymmetry in price transmission relied on some form of non-linearity in the variables of the model. For example, Houck (1977) proposed to decompose transmission between the farm and retail prices in period $t$ (respectively denoted by $f_{t}$ and $p_{t}$ ) by:

$$
\Delta p_{t}=\gamma_{0}+\gamma^{-} D_{t}^{-} \Delta f_{t}+\gamma^{+} D_{t}^{+} \Delta f_{t}+w_{t}
$$

where $D_{t}^{+}$and $D_{t}^{-}$are dummy variables indicating whether the change in the farm price is respectively positive or negative. Equation (1) is linear in the parameters but is clearly non-linear in the variables because the dummy variables are defined as a function of the difference in the farm price. Differences between $\gamma^{-}$and $\gamma^{+}$lead to different magnitude effects of price shocks at the farm level, and were thus interpreted as evidence of APT in magnitude. Conversely, Ward (1982) proposed to estimate the following equation:

$$
\Delta p_{t}=\gamma_{0}+\sum_{j=0}^{M} \gamma_{j}^{-} D_{t-j}^{-} \Delta f_{t-j}+\sum_{j=0}^{M} \gamma_{j}^{+} D_{t-j}^{+} \Delta f_{t-j}+w_{t}
$$


where the individual $\gamma_{j}^{i}(i=-,+; j=1, \ldots, M)$ capture the speed of adjustment, while the sum of the $\gamma_{j}^{i}$ captures the magnitude of adjustment between the farm and retail prices. The literature quickly evolved toward modelling the stochastic properties of the data. Because price series are often found to be non-stationary (Wang and Tomek, 2007), firstdifferencing the data in (1) and (2) was technically correct. However, first-differencing the series can remove information about the common trend observed by prices if they are cointegrated. The framework in (2) can be re-cast in terms of an error correction framework with a long-run equilibrium:

$$
\begin{aligned}
& \Delta p_{t}=\gamma_{0}+\sum_{j=0}^{M} \gamma_{j}^{-} D_{t-j}^{-} \Delta f_{t-j}+\sum_{j=0}^{M} \gamma_{j}^{+} D_{t-j}^{+} \Delta f_{t-j}+\tau^{+} I_{t-1}^{+} w_{t-1}+\tau^{-} I_{t-1}^{-} w_{t-1}+\varepsilon_{t} \\
& w_{t}=p_{t}-\alpha-\beta f_{t}
\end{aligned}
$$

where $I_{t-1}^{+}$and $I_{t-1}^{-}$are dummy variables indicating whether the deviation in the long-run equilibrium between the farm and retail prices (denoted by $w_{t-1}$ ) is positive or negative. The coefficients $\tau^{+}$and $\tau^{+}$measure the speed to which prices return to their equilibrium. Naturally, a test of asymmetry in the speed of price transmission is a test of the null hypothesis $\tau^{+}=\tau^{-}$. Meyer and von Cramon-Taubadel (2004) note that the cointegration framework in (3) can only be used to investigate asymmetry in the speed of adjustment because APT in magnitude implies different long-run responses between negative and positive episodes of price shocks, and thus is inconsistent with prices that are linearly cointegrated.

This last observation is precisely the point that the current paper investigates. As detailed in the introduction, most of the empirical applications investigate a "speed-ofadjustment" type of asymmetry. It is however not difficult to construct an example in which one should not expect prices to be linearly cointegrated. Consider the following 
stylized model of price transmission. Food retailers produce a ready-to-consume commodity using a farm product and other inputs whose prices are exogenous to the industry. Assume that the country is small and can import the farm product at a constant world price. To simplify the nature of competition in the retail industry, it is assumed that while food retailers can shop the globe for primary product supplies, consumers only travel to a few local grocery stores to purchase food. Hence, we will assume that domestic consumers are totally captive and cannot have products shipped in from other regions. This allows for some control over prices by food retailers. Denote the marginal cost function of retailers by: $M C(Q, f)$, where $Q$ denotes output of a retailer and $f$ is the world price of the farm input. Suppose there are $N$ identical retail firms. In period $t$, profit maximizing behaviour yields:

$$
\frac{p_{t}-M C\left(Q_{t}, f_{t}\right)}{p_{t}}=\frac{\lambda_{t}}{N \varepsilon}
$$

where $p_{t}$ is the retail price at time $t, \varepsilon$ is the price elasticity of consumers' demand (assumed constant) and $\lambda_{t}$ is a conjectural variation parameter which measures the anticipations of a firm with respect to changes in output. Under perfect competition, $\lambda_{t}=0$, while perfect collusion among the $N$ firms implies that $\lambda_{t}=N$. Solving (5) yields the retail price:

$$
p_{t}^{*}=\frac{M C\left(Q_{t}^{*}, f_{t}\right)}{\left(1-\lambda_{t} /(N \varepsilon)\right)}
$$

To analyze the price transmission issue, first assume that marginal cost is independent of output and thus $M C\left(Q_{t}^{*}, f_{t}\right)=\beta_{0} f_{t}^{\beta_{1}}$. Substituting marginal cost into (6) yields:

$$
\ln p_{t}^{*}=-\ln \beta_{0}+\beta_{1} \ln f_{t}+w_{t}
$$


where $w_{t} \equiv-\ln \left(1-\lambda_{t} /(N \varepsilon)\right)$ is a collection of unobserved terms from the econometrician's perspective. If the (logarithmic transformation of) $f_{t}$ and $p_{t}$ follow a random walk, equation (7) represents a stable relationship only if the error term is stationary. Hence, a strategy would be to estimate (7) with standard OLS and test the null hypothesis of a unit root in the residuals (no-cointegration). If the null hypothesis is rejected, we can conclude that prices are tied by a log-linear relationship and that adjustment towards this equilibrium is symmetric in the sense that positive deviations will drift back to the equilibrium at the same speed as negative deviations.

The model of asymmetric price transmission in (3) and (4) can also be analyzed in the context of the simple stylized model in (7) by assuming that the adjustment path towards the equilibrium can be represented by:

$$
\Delta w_{t}=\rho_{0}+\rho^{+} I_{t-1}^{+} w_{t-1}+\rho^{-} I_{t-1}^{-} w_{t-1}+v_{t}
$$

Obviously, the dynamics in the context of the stylized model above must come from $\lambda_{t}$ which in essence summarizes information about the nature of competition in the industry.

The log-linear long-run relationship is conditional on the assumptions made with regards to the marginal cost. In a more general framework, a different functional form for marginal cost could yield a non-linear relationship between the farm and retail prices. ${ }^{1}$ This non-linearity is a necessary condition for the existence of APT in magnitude; i.e. through the response in the output price made conditional on the direction of the change in the input price. In the context of cointegration, a non-linear long-run relationship between prices can imply APT in magnitude, while APT in speed would be captured by a nonlinear adjustment process towards the long-run equilibrium. It must be noted that nonlinearity in itself is not sufficient to have APT. For example, a standard threshold model in

\footnotetext{
${ }^{1}$ McCoriston, Morgan and Rayner (2001) derive the price transmission elasticity between different levels of the market under general conditions with respect to the cost structure of downstream firms and competition among firms.
} 
the form of $\ln p_{t}^{*}=\alpha_{0}+\alpha_{1}^{+} I_{t-1}^{+} \ln f_{t}+\alpha_{1}^{-} I_{t-1}^{-} \ln f_{t}+w_{t}$ admits symmetry in the magnitude of price transmission under certain conditions with respect to regime definitions (i.e. with respect to the definition of $I_{t-1}^{+}$and $\left.I_{t-1}^{-}\right)$. The focus of the paper is on disentangling nonlinearity in the long-run price relationship from non-linearity in the adjustment towards equilibrium.

The empirical application focuses on the U.S. hog/pork supply chain. The first step in the overall estimation strategy will be to test the null hypothesis of a (log-)linear relationship between the farm and retail prices using Choi and Saikkonen (2004)'s testing procedure. The rejection of the null hypothesis leads to the Smooth Transition Cointegration (STC) model of Saikkonen and Choi (2004) to estimate the long-run relationship between farm and retail prices. Under the STC framework, the relationship is assumed to change smoothly according to some transition mechanism which is function of prices (e.g. a logistic function).

One drawback of the method is that the limit distribution theory of the parameters is derived under the assumption that the error terms form a stationary process (i.e., under the hypothesis of cointegration). It would thus be useful to test for cointegration before carrying inference about the parameters; but the distributional theory of such a test has yet to be fully developed. ${ }^{2}$ The cointegration framework is however consistent with many forms of short-run dynamics. The auto-regressive process of the residuals reveals information about the speed at which variables return to their long-run equilibrium. Hence, numerous authors focus on the behaviour of the residuals to detect asymmetry in the speed

\footnotetext{
${ }^{2}$ Choi and Saikkonen (2007) suggest a procedure similar to the Kwiatkowski, Phillips, Schmidt and Shin (KPPS, 1992)'s stationarity test to investigate whether the variables are cointegrated under the smooth transition cointegration framework. Breitung (2001) proposes rank tests for non-linear cointegration but the underlying assumptions of the procedure significantly reduce the power of these tests in the current framework.
} 
of price transmission. ${ }^{3}$ A two-step procedure, in the spirit of Balke and Fomby (1997)'s threshold cointegration model, is used to investigate cointegration and non-linear adjustment towards equilibrium. First, standard unit root tests can investigate the probability of rejecting the null hypothesis of a unit root against the alternative hypothesis of stationarity. In a second step, different testing procedures (developed by Luukkonen, Saikkonen and Teräsvirta, 1988; Tsay, 1989; and Hansen, 1999) are used to investigate different types of non-linearities to uncover potential APT in speed.

Figure 1 illustrates the sequence of the procedure to attempt sorting out asymmetry in speed and magnitude. The first step involves testing non-linearity in the relationship between prices. If the null hypothesis of linearity is rejected, the STC cointegration model can be estimated; and the predicted residuals are used to implement the two-step methodology described above. The left hand-side matrix at the bottom of Figure 1 illustrates the starting point of most studies dealing with asymmetric price transmission

It is found that the long-run relationship between the U.S. hog and pork prices is non-linear. A unit root bootstrap procedure (Parker et al., 2006) applied to the residuals of the non-linear cointegrating vector suggests that the series is stationary. The null hypothesis that the residuals follow a linear process against various alternatives of nonlinearity is not rejected. The same two-step procedure was applied to the residuals generated by a linear cointegrating vector estimated by Ordinary Least Squares (OLS). The evidence is less conclusive in this case as one of the tests rejects the null of linearity in favour of smooth transition autoregressive behaviour.

The above finding raises the possibility that the failure to account for non-linearity in the cointegrating vector may have an impact on the probability of detecting non-

\footnotetext{
${ }^{3}$ Only an error correction model would fully characterize the short-run dynamics of the process. Escribano and Mira (2002) and Saikkonen (2005) provide guidance to specify an error correction model under nonlinear adjustment towards the equilibrium. It is unknown whether there exist a representation of an error correction model when the long-run relationship is non-linear.
} 
linearity in the adjustment towards equilibrium. A short Monte Carlo investigation is proposed to investigate the properties of the two-step procedure under non-linear cointegration Our results emphasize the importance of first investigating potential nonlinearity in the long-run relationship between the variables when studying price transmission.

\section{Data}

Monthly data on the hog (farm) price and the pork (retail) price were collected from the ERS-USDA dataset on meat price spreads covering the period January 1980 to August 2006. The farm price is the AMS 51-52\% base-lean-hog price. The retail price is a weighted average of the retail prices for specific pork cuts as reported by the Bureau of Labor Statistics (BLS). The farm and retail prices were weighted by the U.S. Consumer Price Index (CPI) to remove a potential general inflation trend, and are thus expressed in 1982-84 dollars per pound. Figure 2 presents the pattern of farm and retail prices in real terms from January 1980 to August 2006. The North American crisis in the hog/pork sector in the second half of 1998 is clearly apparent in the figure. The monthly hog price declined by more than 63 percent between June 1998 and December 1998, while the decline in the retail price was less than 3 percent over the same period. The retail price also appears less volatile in the second half of the sample than the farm price. Figure 3 presents the relationship between the logarithmic transformation of the monthly average of the farm and retail prices in the U.S. hog/pork sector. A swift visual inspection of the data in Figure 3 reveals the presence of some downward stickiness in the retail price for low values of the farm price. For intermediate to large values of the farm price, the retail and farm prices seem positively correlated. The two lowest values for the farm price represent the November and December 1998 observations and appear to be outliers when analyzed in the context of the linear trend plotted in Figure 3. 
Before estimating the relationship between retail and farm prices, the stochastic properties of the data needs to be assessed. The unit root bootstrap procedure of Parker, Paparoditis and Politis (2006) was also used to investigate if the series are integrated of order one. They propose a residual-based bootstrap procedure that has overwhelmingly better power in small samples than the usual asymptotic tests which tend to under reject the null hypothesis of a unit root (Maddala and Kim, 1998). While the present dataset in this study is not small compared to other well-known applications in price transmission (e.g., Abdulai, 2002; Serra and Goodwin, 2003), the procedure nevertheless offers significant improvements over asymptotic theory because it does not rely on a specific data generating process.

The stationary bootstrap procedure is quite simple. Consider a time series $X_{t}$ $(t=1, \ldots, T)$ and define the centered (Horowitz, 2001) residuals $\hat{v}_{t}=X_{t}-\hat{\alpha}-\hat{\rho} X_{t-1}$ where $\hat{\alpha}$ and $\hat{\rho}$ are the Ordinary Least Squares (OLS) estimates of the autoregressive process: $X_{t}=\alpha+\rho X_{t-1}+v_{t}$. The idea is to sample blocks of residuals whose length are randomly selected using a geometric distribution with parameter $q$. A bootstrap sample is formed by setting the first observation of the bootstrap sample to its sample value $\left(X_{1}^{*}=X_{1}\right)$. The second observation in the bootstrap sample will be $X_{2}^{*}=X_{1}^{*}+v_{2}^{*}$, where $v_{2}^{*}=\hat{v}_{t}$. The following observation will be: $X_{3}^{*}=X_{2}^{*}+v_{3}^{*}$; where $v_{3}^{*}=\hat{v}_{t+1}$ with probability $1-q$ or $v_{3}^{*}=v_{s}^{*}$ with probability $q$ and $s \in[1, \ldots, T]$. Using the bootstrap sample, the OLS estimate $\hat{\rho}^{*}$ is computed. This procedure is repeated $B$ times and the empirical rejection probabilities are computed. In practice, there is no theoretical basis to select the parameter of the geometric distribution. We experimented with a few different parameters to find it did not change the qualitative nature of the results and chose to report the results for 
$q=0.1$ with $B=2000$. Table 1 reports the $p$-values of the null hypothesis of a unit root. The evidence suggests that the null hypothesis of a unit root cannot be rejected.

Granger (1980) showed that a sum of AR(1) processes can result in a long-memory process. Given that monthly prices in the dataset can be thought of a weighted sum of weekly or daily prices, the previous unit root tests may uncover what is really persistence of shocks in stationary processes. More generally, a series can be considered fractionally integrated of order $d$, with $d=0$ and $d=1$ being two special cases. There are two broad methods to compute the value of $d ; i$ ) log-periodogram regression; and ii) Local Whittle (LW) estimation (Shimotsu and Phillips, 2005). Shimotsu (2006) proposes a feasible exact LW estimator in which the unknown mean of the series is estimated as a combination of the sample average and the initial observation depending on the value of the parameter $d$. A two-step estimator is also proposed to eliminate some of the problems associated with the consistency of the feasible exact LW for some intervals of $d$. The $95 \%$ confidence intervals of the estimates of $d$ for each series are presented in table 1 . They are computed based on the asymptotic distribution of each estimator. All the intervals exclude the point $d=0.5$ and thus the data is said to be non-stationary. Despite that non-stationarity does not imply integration of order one, the overall evidence suggests with some confidence that the variables in the model can be considered $I(1)$ processes.

\section{Non-linear cointegration}

The next step in the empirical investigation is to estimate the relationship between the farm and retail prices. As mentioned before, most studies attempt to detect a non-linearity in the adjustment path towards the long-run equilibrium. As it is well known, two integrated series are said to be cointegrated if there exists a stable attractor between the series such that they drift to a long-run equilibrium (Engle and Granger, 1987). Residual-based tests of cointegration involve estimating a linear cointegrating vector and then using the estimated 
residuals to test for cointegration. Under the null hypothesis of no-cointegration, the residuals follow a random walk and unit root tests can be applied although the distribution of the test statistic must be adapted accordingly. In case a non-linear adjustment path toward equilibrium is suspected, Balke and Fomby (1997) suggest testing for threshold cointegration in a roundabout way; by first testing the null hypothesis of (linear) nocointegration, and second testing for non-linearity in the residuals of the cointegrating vector. $^{4}$

The present approach is based on the Smooth Transition Cointegration (STC) framework of Saikkonen and Choi (2004). Let $f_{t}$ and $p_{t}$ be the farm and retail prices in month $t$ respectively. Under the hypothesis of STC, the relationship between the two variables is:

$$
p_{t}=\alpha+\eta g\left(f_{t}-c ; \gamma\right)+\beta f_{t}+\delta f_{t} g\left(f_{t}-c ; \gamma\right)+u_{t} ; \quad t=1, \ldots, T
$$

where $u_{t}$ is a mean zero stationary error term and $g\left(f_{t}-c ; \gamma\right)$ is a transition function. This transition function can take different forms, but here a logistic function is assumed:

$$
g\left(f_{t}-c ; \gamma\right)=\left[1+\exp \left\{-\gamma\left(f_{t}-c\right)\right\}\right]^{-1}
$$

The implications for the relationship between the two prices defined by the framework laid out in (9) and (10) are straightforward. The transition function is bounded between zero and one; hence the slope of the regression in (9) (which is the price transmission elasticity) varies from $\beta$ to $\beta+\delta$. As the farm price increases and gets further away from the threshold level defined by the parameter $c$, the slope takes a value close to $\beta+\delta$. Conversely, as the farm price decreases, the slope assumes a value close to

\footnotetext{
${ }^{4}$ Enders and Siklos (2001) propose to test non-linearity in the residuals of the cointegration vector using threshold behaviour as the alternative hypothesis. The drawback of this approach is that there are no workable approaches to derive a general limiting distribution of this test because the threshold parameters are not identified under the null. Seo (2006) proposes a sup-Wald statistic in the spirit of Davies (1987) to deal with this problem, but the procedure is strictly valid under the assumption that the cointegrating relation is known.
} 
$\beta$. A similar argument can be made for the constant in the regression. Note that there are possible variants to (9). The transition function need not be the same for the constant and the slope of the regression and the transition function can have a higher order (van Dijk, Teräsvirta and Franses, 2002). The parameter $\gamma$ has an important role in that it dictates the smoothness in the transition between the regression coefficients. As $\gamma$ increases, the change in the transition function from zero to one becomes almost instantaneous when $f_{t}=c$; hence the non-linearity in the prices will be more visible the higher is the value of $\gamma$. In the limit case where $\gamma$ tends to infinity, the model reduces to a standard threshold model. When $\gamma=0$, the price transmission model shrinks to a linear relationship.

Because non-linear models in general are designed to be flexible, it is not unusual for data-mining to occur. Hence, it is important to first test for non-linearity in the relationship between the farm and retail prices before proceeding with the estimation. Choi and Saikkonen (2004) developed non-linearity tests that can be applied in the context of STC. Ideally, one would like to test the null hypothesis $\gamma=0$ directly from (9); but the test is complicated by the fact that some parameters are not identified under the null. They circumvent the problem associated with the presence of nuisance parameters by replacing the transition function with a Taylor approximation. In the current context, the testing procedure involves estimating the following auxiliary regression using OLS:

$$
p_{t}=\alpha+\eta b \gamma\left(f_{t}-c\right)+\beta f_{t}+\delta f_{t} b \gamma\left(f_{t}-c\right)+\sum_{j=-K}^{K} \pi_{j} \Delta f_{t-j}+v_{t} ; \quad t=1, \ldots, T
$$

where $b \equiv \partial g(z) /\left.\partial z\right|_{z=0}$ and $v_{t}$ is an iid shock. Following Saikkonen (1991), leads and lags are used in (11) to correct for potential endogeneity and the resulting correlation between the regressors and the error term under the hypothesis of cointegration. Equation (11) can be rewritten in compact form as: 


$$
p_{t}=\omega+\psi f_{t}+\theta f_{t}^{2}+\sum_{j=-K}^{K} \pi_{j} \Delta f_{t-j}+v_{t} ; \quad t=1, \ldots, T
$$

where $\omega \equiv \beta-\eta b \gamma c, \psi \equiv \beta+\eta b \gamma-\delta b \gamma c$ and $\theta \equiv \delta b \gamma$.

The LM test statistic of the null hypothesis of linearity $(\theta=0)$ is $\tau_{1}=\hat{\theta}\left[\hat{\sigma}_{e}^{2}\left(M^{-1}\right)_{x x}\right]^{-1} \hat{\theta}$; where $\hat{\theta}$ is the OLS estimate of $\theta$ in (12), $\hat{\sigma}_{v}^{2}$ is the variance of the sample residuals based on the latter estimator with the restriction $\theta=0$ and $\left(M^{-1}\right)_{x x}$ is the element of the inverse of the sample moment matrix associated with $f_{t}^{2}$. The statistic follows a chi-squared distribution with one degree of freedom.

Choi and Saikkonen (2004) note that the previous test can have low power to detect non-linearity if $\delta$ is small. Hence, they devised another test using a third degree Taylor approximation that yields the auxiliary regression:

$$
p_{t}=\tilde{\omega}+\tilde{\psi} f_{t}+\theta_{1} f_{t}^{2}+\theta_{2} f_{t}^{3}+\sum_{j=-K}^{K} \pi_{j} \Delta f_{t-j}+\tilde{v}_{t} ; \quad t=1, \ldots, T
$$

The null hypothesis of linearity is $\theta_{1}=\theta_{2}=0$ and the LM statistic is: $\tau_{2}=\hat{\boldsymbol{\theta}}\left[\hat{\sigma}_{e}^{2}\left(M^{-1}\right)_{x x}\right]^{-1} \hat{\boldsymbol{\theta}}$; where $\hat{\boldsymbol{\theta}} \equiv\left[\begin{array}{ll}\theta_{1} & \theta_{2}\end{array}\right]^{\prime}$ and $\left(M^{-1}\right)_{x x}$ includes the elements associated with $\left[\begin{array}{ll}f_{t}^{2} & f_{t}^{3}\end{array}\right]^{\prime}$ of the inverse of the sample moment matrix. The distribution of the $\tau_{2}$ statistic follows a chi-squared distribution with 2 degrees of freedom. Table 2 presents the $\tau_{1}$ and $\tau_{2}$ statistics for $K=1,2,3$. Both tests strongly reject the null hypothesis of linearity for all values of $K$.

Given that the null hypothesis of linearity is rejected, the estimation of the model in (9) is carried out in two stages. First, the Non-Linear Least Squares (NLLS) estimates of the model defined in (9) and (10) are computed. While these estimates are consistent, they are inefficient for the same reasons that generally apply to a linear cointegration model. As 
noted before, the error term of the cointegration equation in (9) might be correlated with the regressor $\left(f_{t}\right)$ and thus $K$ lags and leads of the independent variable are used to purge the potential correlation (Saikkonen and Choi, 2004). ${ }^{5}$ Hence, the cointegrating regression is written as:

$$
p_{t}=\alpha+\eta g\left(f_{t}-c ; \gamma\right)+\beta f_{t}+\delta f_{t} g\left(f_{t}-c ; \gamma\right)+\sum_{j=-K}^{K} \pi_{j} \Delta f_{t-j}+e_{t} ; \quad t=1, \ldots, T
$$

Let the estimates of the first stage NLLS applied to (9) and (10) be represented by the vector $\hat{\mathbf{\Omega}} \equiv\left[\begin{array}{llllll}\hat{\gamma} & \hat{c} & \hat{\alpha} & \hat{\eta} & \hat{\beta} & \hat{\delta}\end{array}\right]^{\prime}$. The second stage least squares estimator is:

$$
\left[\begin{array}{c}
\tilde{\boldsymbol{\Omega}} \\
\tilde{\boldsymbol{\pi}}
\end{array}\right]=\left[\begin{array}{c}
\hat{\boldsymbol{\Omega}} \\
0
\end{array}\right]+\left(\mathbf{q}^{\prime} \mathbf{q}\right)^{-1} \mathbf{q}^{\prime} \hat{\mathbf{u}}
$$

where $\hat{\mathbf{u}}$ is the vector of predicted residuals from the first stage NLLS procedure, $\mathbf{q}_{t} \equiv\left[\begin{array}{ll}\boldsymbol{\Phi}^{\prime} & \Delta \mathbf{f}_{t}^{\prime}\end{array}\right]^{\prime} \quad$ with $\quad \boldsymbol{\Phi} \equiv\left[\begin{array}{llllll}\hat{\delta} f_{t} \frac{\partial g\left(f_{t}-\hat{c} ; \hat{\gamma}\right)}{\partial \gamma} & \hat{\delta} f_{t} \frac{\partial g\left(f_{t}-\hat{c} ; \hat{\gamma}\right)}{\partial c} & 1 & g\left(f_{t}-\hat{c} ; \hat{\gamma}\right) & f_{t} & g\left(f_{t}-\hat{c} ; \hat{\gamma}\right) f_{t}\end{array}\right]^{\prime}$ and $\Delta \mathbf{f}_{t} \equiv\left[\begin{array}{lll}\Delta f_{t-K} & \ldots & \Delta f_{t+K}\end{array}\right]^{\prime}$. The second stage estimator in (15) is called a GaussNewton (GN) estimator. The second term on the right hand-side of (15) is the result of regressing the error terms of the first-stage NLLS procedure on the vector $\mathbf{q}$ which includes the gradient evaluated at the optimal solution and leads and lags of the independent variable.

Saikkonen and Choi (2004) present a Monte Carlo simulation to investigate the properties of their estimators. They find that the efficiency gain associated with the twostage estimator is positively correlated with the contemporaneous correlation between the error term $(u)$ and the regressor. They also investigated the statistical properties (bias and

\footnotetext{
${ }^{5}$ Saikkonen and Choi (2004) argue that the number of leads and lags in (14) has very little effect on the size of the testing procedure as long as the number of leads and lags are selected to increase at an appropriate rate with the sample size. Chang and Park (2003) suggest another possibility which is to use auxiliary regressions to purge correlation between the error terms and regressors in a preliminary step before using NLLS in a second stage.
} 
efficiency) of a second iteration involving the second-stage GN estimator as the starting point estimates in (15). They found that this second iteration achieves a significantly lower root mean-squared error than the two-stage GN and NLLS estimators; thus suggesting that an additional iteration of the second-stage procedure defined in (15) might be desirable. The results reported below follow this approach. The first-stage NLLS estimates at the start of the iteration process are not reported below but their standard errors were quite large; implying that there was a strong correlation between the error terms of the cointegration equation and the farm price. In what follows, a value of one was selected for the parameter $K$ and unreported results suggest that increasing the value of this parameter did not improve the accuracy of the estimation procedure.

The general model in (14) was first estimated without any restrictions on the parameter space because it is difficult to pinpoint a specific functional form for the markup model implied by (6). The estimate of the smoothness parameter in the transition function was excessively large (276.03) with an even larger standard error. The problem is that the model converges to a threshold model as the parameter $\gamma$ increases, and thus one needs many observations in the neighbourhood of $c$ to get a precise estimate of $\gamma$ (van Dijk, et al. 2002; Saikkonen and Choi, 2004). While this does not constitute ground in itself to reject the model, the model was re-estimated imposing the restriction that the constant term was not switching according the level of the farm price $(\eta=0)$. This restriction significantly improved the precision in the estimates of the other parameters. Table 3 presents the (iterated) GN estimates of the cointegration model along with their standard errors. Conventional inference is difficult to carry because the null hypothesis that the parameters $\gamma, c$ and/or $\delta$ are zero involves nuisance parameters. The distribution of the linear parameters is however asymptotically normal (Saikkonen and Choi, 2004). The 
standard error of $\alpha$ implies that the constant is statistically significant and estimated with precision. The slope parameter $(\beta)$ is also positive with a very small standard error.

To provide a little more intuition on the significance of the STC model, Figure 4 presents the predicted relationship between the U.S. hog/pork prices following the STC model as well as the linear cointegration model. The non-linearity seems to be mostly associated with a lower bound on the value of the retail price. The high retail price values (which are observed incidentally early in the sample) are accompanied by similarly high farm prices. The elasticity of price transmission is higher under the STC model than under the linear model when prices are high. However, unlike the linear model, the STC model predicts some sort of retail price stickiness when farm prices are low. The impact of a change in the farm price is conditional on the level of the farm price. The asymmetry in magnitude is especially apparent in midpoints of the sample. Positive shocks in the farm price will have a larger impact on retail prices than negative shocks.

A number of theories could explain downward price stickiness. One is that when input prices fall, the previous output price offers a natural focal point for oligopolistic sellers. In response to a decrease in costs, a downstream firm might choose not to change its price until demand conditions evolve. This variant of the "trigger price" model of Green and Porter (1984) is discussed in Borenstein, Cameron and Gilbert (1997). Under this framework, increases in farm prices trigger retail price increases; while conversely, it is possible to have retail price inertia following negative farm price shocks. It must be stated however that evidence of downward price stickiness must not be interpreted as evidence of imperfectly competitive behaviour because adjustment costs in a perfectly competitive supply chain can also yield this sort of price relationship. However, in light of increased concentration in pork packing activities, the results suggest that further research should investigate potential causes of downward retail price stickiness. 


\section{Non-Linear adjustment towards equilibrium}

The discussion of the previous section hinges on the notion that the two variables are indeed cointegrated. This section investigates the properties of the residuals of the cointegrating regression in (14). Precisely, Balke and Fomby (1997)'s two-step procedure is implemented to study the behaviour of the residuals. The first step involves testing for the presence of a unit root. In case the null hypothesis of a unit root is rejected, the nonlinearity tests of Luukkonen, Saikkonen, and Teräsvirta (1988), Tsay (1989), and Hansen (1999) are computed under the assumption that the error terms are stationary. The right hand-side matrix at the bottom of Figure 1 illustrates the procedure.

The left hand-side column of table 4 reports the results of the stationary bootstrap procedure applied to the fitted residuals of (14). The null hypothesis of no-cointegration is clearly rejected by the data. ${ }^{6}$ As mentioned before, the literature on price transmission documented many times some form of non-linearity in the error-correction process of the linear long-run relationship between the retail and farm prices. Three linearity tests for the residuals $\hat{e}_{t}$ are proposed here. First, the procedure of Luukkonen, Saikkonen, and Teräsvirta (1988, hereafter LST) is implemented to test linearity against the alternative of smooth transition autoregression conditional on whether $e_{t-1} \frac{\geq}{<} 0$. Much like the procedure described to test linearity in the relationship between the farm and retail prices, the procedure involves testing the null hypothesis $\beta_{1}=\beta_{2}=\beta_{3}=0$ for the auxiliary regressions:

$$
\hat{e}_{t}=\beta_{0} \hat{e}_{t-1}+\beta_{1} \hat{e}_{t-1}^{2}+\beta_{2} \hat{e}_{t-1}^{3}+\beta_{3} \hat{e}_{t-1}^{4}+\xi_{t}
$$

\footnotetext{
${ }^{6}$ The evidence generated by the bootstrap procedure must however be interpreted with caution because there are no guarantees that the bootstrap procedure provides a better approximation than the standard asymptotic distribution in the current non-linear cointegration framework.
} 
The statistic is based on the sum of squared residuals under the null and alternative hypotheses and follows a chi-squared distribution with two degrees of freedom.

The two other testing procedures have power against threshold behaviour in the series. Tsay (1989)'s univariate threshold model with $k$ regimes is:

$$
\hat{e}_{t}=\psi^{(j)} \hat{e}_{t-1}+\xi_{t} ; \quad \Upsilon_{j-1}<e_{t-1} \leq \Upsilon_{j} ; \quad-\infty=\Upsilon_{0}<\Upsilon_{1}<\ldots<\Upsilon_{k}=\infty
$$

The parameters $\Upsilon_{j}(j \neq 0, k)$ need to be estimated through sequential least squares. Before estimating (17), we can test the null hypothesis that the model in (17) is linear against the alternative that it follows a univariate threshold specification. Tsay (1989)'s idea is based on the change-point literature in which the regression in (17) is rearranged according to the ascending order of the threshold variable. If the model is linear, the predictive residual of the arranged regression model will be uncorrelated with the regressors, whereas the residuals will not be white noise if a threshold effect is present. In our application, the test statistic is distributed according to a chi-squared distribution with one degree of freedom.

Hansen (1999)'s Self-Exciting Threshold Autoregressive Model (SETAR) with $m$ regimes is also considered. The $\operatorname{SETAR}(m)$ model takes the general form:

$$
\hat{e}_{t}=\mu_{1} \hat{e}_{t-1} I_{1}(\Upsilon)+\ldots+\mu_{m} \hat{e}_{t-1} I_{m}(\Upsilon)+\xi_{t}
$$

where $\Upsilon \equiv\left(\Upsilon_{1}, \ldots, \Upsilon_{m}\right)$ and $I_{j}(\Upsilon)=\Upsilon_{j-1}<\hat{e}_{t-1} \leq \Upsilon_{j}$ is an indicator function. The estimation of (18) is done sequentially. Conditional on the number of regimes and potential threshold values, the OLS estimate of $\boldsymbol{\mu} \equiv\left[\begin{array}{lll}\mu_{1} & \ldots & \mu_{m}\end{array}\right]$ is obtained along with the residual sum of square errors denoted $S_{m}(\Upsilon ; \hat{\boldsymbol{\mu}})$. The estimates of the thresholds are given by: $\hat{\Upsilon}=\arg \min S_{m}(\Upsilon ; \hat{\boldsymbol{\mu}})$. The search for potential threshold values is restricted to the values of $\hat{e}_{t-1}$ that lie between the $\tau$ and $(1-\tau)$ th quantiles $(\tau=0.1$ in the current application). The test of the null hypothesis of a single regime (linearity) against the 
alternative that the process $\hat{e}_{t}$ is described by $m$ regimes is given by: $F_{1 m}=\left(S_{1}\left(\mu_{1}\right)-S_{m}(\hat{\Upsilon} ; \hat{\boldsymbol{\mu}})\right) / S_{m}(\hat{\Upsilon} ; \hat{\boldsymbol{\mu}})$. However, the $F_{1 m}$ statistic is equivalent to maximizing standard $F$ statistics and its distribution is a function of the degree of dependence across the different $F$ tests. Hansen (1999) suggests using either asymptotic approximations or bootstrap in which the distribution of the statistic is simulated under the null hypothesis. We use the latter in what follows with 2,000 replications.

The LST procedure suggests the residuals follow a linear process as the probability of rejecting the null hypothesis of linearity when it is true is larger than 0.1 in the second column of table 4 . The third column of table 4 reports the $p$-value of Tsay's non-linearity test and does not provide evidence of non-linearity in the residuals. Finally, the last column of table 4 presents the $p$-value of the $F_{12}$ test. The $F_{12}$ test does not reject the null hypothesis of one regime in the residuals (linearity) against the alternative of two regimes at a conventional significant level. All three tests suggest that there is no asymmetry in the speed of price transmission.

The above results are somewhat in contradiction with the findings of Goodwin and Harper (2000) who report evidence of asymmetry in the speed of price transmission in the hog/pork supply chain. Notwithstanding the differences in the sample period and data coverage between the current analysis and their study, the main factor is that their test assumes a priori a linear relationship between prices in the hog/pork supply chain. It is thus interesting to investigate whether residuals generated by a linear relationship between farm and retail prices would uncover a similar type of asymmetry. Table 5 presents the same non-linearity tests applied to the fitted residuals of a linear cointegration framework estimated with standard OLS. The purpose is to investigate whether non-linearity in the adjustment process towards the cointegrating vector can be detected if the non-linearity in the magnitude of price transmission is not addressed in the first stage. 
The bootstrap unit root clearly rejects the null hypothesis of a unit root in the fitted residuals; and thus suggests that farm and retail prices are indeed cointegrated. Both the Tsay and Hansen non-linearity tests produce large $p$-values. However, the LST procedure clearly rejects the null hypothesis of linearity in favour of smooth transition in the behaviour of the residuals. This clearly illustrates that failure to account for nonlinearity in the long-run relationship between prices may wrongly lead to the conclusion that the adjustment process towards equilibrium is non-linear.

A Monte Carlo investigation illustrates some of the pitfalls associated with testing for non-linearity in the speed of price transmission. It follows closely the investigation described in Saikkonen and Choi (2004). First, a random walk of length equal to 300 is generated to match the length of our dataset: $x_{t}=x_{t-1}+v_{t}$; where $v_{t}$ is a random error term. The initial value of the series is normalized to zero $\left(x_{0}=0\right)$. Next, the time series $y_{t}$ is generated according to: $y_{t}=\alpha+\beta x_{t}+\delta x_{t} g\left(x_{t} ; \gamma, c\right)+u_{t}$; where $\alpha=\beta=1$ and the transition function $g\left(x_{t} ; \gamma, c\right)$ is defined as in (10) in Author (2007) with $\gamma=1, c=0$. To determine whether there are significant size distortions in the non-linearity tests applied to the residuals, the following process is assumed for the error term of the long-run relationship between $x_{t}$ and $y_{t}: u_{t}=0.75 u_{t-1}+\varepsilon_{t}$. To evaluate the power of the tests, the error sequence is generated by $u_{t}=0.8 I_{\left(u_{t-1}>0\right)} u_{t-1}+0.4 I_{\left(u_{t-1}<0\right)} u_{t-1}+\varepsilon_{t}$; where $I_{(\cdot)}$ is the indicator function of the lagged residual. In both cases, the following distributional assumptions are made: $\left[\begin{array}{l}\varepsilon_{t} \\ v_{t}\end{array}\right] \sim N\left(\left[\begin{array}{l}0 \\ 0\end{array}\right],\left[\begin{array}{cc}1 & 0.5 \\ 0.5 & 1\end{array}\right]\right)$.

Armed with the simulated series, the regression $y_{t}=\beta_{0}+\beta_{1} x_{t}$ is estimated by OLS and the predicted residuals $\hat{\mathbf{u}}^{L}$ are saved in a vector. Next, the Gauss-Newton estimates of 
the STC equation are obtained and the residuals stored in the vector $\hat{\mathbf{u}}^{S T C}{ }^{7}$ The Monte Carlo investigation begins at the second stage of the Balke-Fomby procedure. ${ }^{8}$ The nonlinearity tests of Hansen (1999), Tsay (1989) and Luukkonen, Saikkonen, and Teräsvirta ( LST, 1988) are applied to the $\hat{u}_{t}^{L}$ and $\hat{u}_{t}^{S T C}$ series.

The overall experiment is repeated a thousand times and the number of rejections for each investigation is recorded using significance levels, 10, 5 and 1 percent for both tests. Table 6 presents the size of the two tests when the estimated cointegrating vector is: a) estimated by a linear model; or b) estimated using the STC framework. There are striking differences between the two cases. The size of all three tests is very poor when the residuals are generated by a linear cointegrating vector (with the LST procedure recording the worst performance). Overall, there is a significant probability that the Balke-Fomby procedure uncovers non-linear adjustment towards equilibrium although the non-linearity resides in the long-run price relationship (i.e. is induced by the non-linear cointegrating vector). Conversely, the Hansen and Tsay tests severely over-reject the null hypothesis of linearity when the residuals are generated by the STC framework. The size distortions are also important when the LST procedure is implemented.

Table 7 presents the power of the two tests under the assumption that the error correcting process of the non-linear cointegrating vector follows a threshold process. First, the Hansen procedure has relatively better power than the Tsay tests in that it leads to more correct rejection of the null hypothesis of linearity for the error correction terms. At a higher level of significance (10 percent), the power of these two tests is better when the

\footnotetext{
${ }^{7}$ Because the estimation is complicated when the parameter $c$ is far away from the median, we made sure that the value of the parameter $c$ was included between the $10^{\text {th }}$ and $90^{\text {th }}$ percentiles of the $x_{t}$ series. If the value of $c$ was outside of this range, the simulated series was thrown out and another series was generated.

${ }^{8}$ The power of the bootstrap unit root test could also be investigated in future work because the linear estimation procedure when the data generating process is non-linear could lead to less frequent rejections of the false null hypothesis.
} 
residuals from the STC model are used compared to the residuals generated by a linear cointegrating vector. But overall, the power of these tests is quite disappointing. The Tsay and Hansen non-linearity tests under the STC framework have power similar to what Balke and Fomby (1997) uncovered when investigating the performance of their two-step procedure under the assumption that the variables were linearly cointegrated. The LST procedure has better power than the other tests, despite the latter being devised with threshold behaviour as the alternative hypothesis.

The main practical implication of the findings is that it is important to first test for linearity in the long-run relationship between the variables before proceeding to analyze the adjustment towards equilibrium. Unfortunately, the stochastic properties of the variables limit the options available to test the null hypothesis that the relationship between prices is linear; and some of the most powerful testing procedures often require stationary regressors (see for example Dahl, 2002).

\section{Conclusion}

Asymmetric Price Transmission (APT) is an increasingly disputed issue in both academic and practitioners' spheres due to, among other things, concerns that concentration in upstream and downstream industries may hold back competitive pricing behaviour. A number of empirical studies confirm certain forms of asymmetry in the speed of price transmission. While prices in upstream and downstream markets converge to their longrun equilibrium at different speed depending on the extent of the market disturbances, the usual idea is that prices are tied by a linear long-run relationship. While there are a few studies documenting potential asymmetries in the magnitude of price transmission, no studies tried to extend the concept of linear cointegration between prices to a non-linear framework. 
The objective of the paper is to study whether it is possible to separate out nonlinearity in the long-run farm-to-retail price relationship from non-linearity in the adjustment towards equilibrium. The smooth transition cointegration model of Saikkonen and Choi (2004) is applied to estimate the long-run relationship between farm and retail prices because these series are integrated of order one. The relationship between the two prices is assumed to change smoothly according to a logistic function. This procedure also leads to a non-linearity test using first and third-order Taylor approximations. The cointegration framework is also consistent with many forms of short-run dynamics. Hence, Balke and Fomby (1997)'s two-step procedure is used to investigate non-linear adjustment towards the equilibrium. The procedure consists of first testing the null hypothesis of a unit root, and subsequently testing the null hypothesis of linearity in the residuals generated by the cointegration model.

There is overwhelming evidence that the farm-to-retail price relationship between the U.S. hog and pork meat prices is non-linear. The unit root bootstrap procedure applied to the residuals of the non-linear cointegrating vector rejects the null hypothesis of nocointegration. The null hypothesis that the stochastic process of the residuals is linear against the alternative of threshold behaviour is also not rejected. The same two-step procedure was applied to the residuals generated by a linear cointegrating vector and estimated with standard ordinary least squares. Interestingly, the procedure clearly rejects the null hypothesis of linearity in the residuals. While the evidence suggests there is a strong non-linearity in the long-run relationship between farm and retail prices, there is less evidence in favour of asymmetry in the magnitude of price transmission because the long-run relationship approaches a standard two-regime (threshold) model.

The findings in the paper should not be interpreted as evidence of imperfect competition in the U.S. hog/pork supply chain, but should incite more research into the 
determinants of asymmetric price transmission. In particular, estimating structural models of price transmission allowing for inventories and adjustment costs would broaden the understanding of asymmetry in price transmission. Finding evidence of downward price stickiness in agricultural supply chains is certainly not unique as others documented slower responses of retail prices following negative shocks to farm prices. In standard models however, decreases in the farm prices are eventually passed along to consumers through lower prices. The results of the non-linear cointegration framework suggest that lower costs may not be passed onto consumers, questioning the efficiency of price transmission in the U.S. hog/pork supply chain.

\section{References}

Abdulai, A. 2002. "Using Threshold Cointegration to Estimate Asymmetric Price Transmission in the Swiss Pork Market". Applied Economics 34: 679-687

Aparicio, F., A. Escribano, and A. Sipols. 2006. "Range Unit-Root (RUR) Tests: Robust against Nonlinearities, Error Distributions, Structural Breaks and Outliers". Journal of Time Series Analysis 27: 545-576.

Author. 2007. "AJAE Appendix: Disentangling Asymmetry in the Magnitude and Speed of Price Transmission in the U.S. Hog/Pork Supply Chain". Unpublished manuscript. Available at: http://agecon.lib.umn.edu/.

Azzam, A. M. 1999. "Asymmetry and Rigidity in Farm-to-Retail Price Transmission". American Journal of Agricultural Economics 81: 525-533.

Balke, N. S., and T. Fomby. 1997. "Threshold Cointegration". International Economic Review 38: 627-643

Borenstein, S., A. C. Cameron, and R. Gilbert. 1997. "Do Gasoline Prices Respond Asymmetrically to Crude Oil Price Changes". Quarterly Journal of Economics 112: 305339.

Breitung, J. 2001. "Rank Tests for Nonlinear Cointegration". Journal of Business and Economic Statistics 19: 331-340.

Chang, Y., and J.Y. Park. 2003. "Index models with Integrated Time Series". Journal of Econometrics 114: 73 - 106.

Chavas, J-P., and A. Mehta. 2004. "Price Dynamics in a Vertical Sector: The Case of Butter”. American Journal of Agricultural Economics 86: 1078-1093. 
Cheung, Y-W., and K. S. Lai. 1995a. "Lag Order and Critical Values of the Augmented Dickey-Fuller Test". Journal of Business \& Economic Statistics 13: 277-280.

Cheung, Y-W., and K. S. Lai. 1995b. "Lag Order and Critical Values of a Modified Dickey-Fuller Test”. Oxford Bulletin of Economic and Statistics 13: 277-280.

Choi, I., and P. Saikkonen. 2004. "Testing Linearity in Cointegrating Smooth Transition Regressions". Econometrics Journal 7: 341-365.

Choi, I., and P. Saikkonen. 2007. “Tests for Non-Linear Cointegration”. Working Paper, University of Helsinki.

Dahl, C. M. 2002. "An Investigation of Tests for Linearity and the Accuracy of Maximum Likelihood based Inference using Random Fields". The Econometrics Journal 5: 263284.

Davies, R. B., 1987. "Hypothesis Testing when a Nuisance Parameter is Present Only Under the Alternatives". Biometrika 74: 33-43.

Enders W., and P. L. Siklos. 2001. "Cointegration and Threshold Adjustment". Journal of Business and Economics Statistics 19: 166-176.

Engle R. F., and C. W. J. Granger. 1987. "Co-integration and Error Correction: Representation, Estimation, and Testing”. Econometrica 55: 251-276.

Escribano, A., and S. Mira. 2002. "Nonlinear Error Correction Models". Journal of Time Series Analysis 23: 509-522.

Frey, G., and M. Manera. 2007. "Econometric Models of Asymmetric Price Transmission”. Journal of Economic Surveys 21: 349-367.

Goodwin, B.K., and D. C. Harper. 2000. "Price Transmission, Threshold Behaviour and Asymmetric Adjustment in the U.S. Pork Sector". Journal of Agricultural \& Applied Economics 32: 543-553.

Goodwin, B. K., and M. T. Holt. 1999. "Asymmetric Adjustment and Price Transmission in the U.S. Beef Sector”. American Journal of Agricultural Economics 81: 630-637.

Granger, C. W. J. 1980. "Long Memory Relationships and the Aggregation of Dynamic Models". Journal of Econometrics 14: 227-238.

Green, E. J., and R. H. Porter. 1984. "Non-cooperative Collusion under Imperfect Price Information”. Econometrica 52: 87-100.

Hahn, W. 2004. Beef and Pork Values and Price Spreads Explained. Electronic outlook report from the Economic Research Service (ERS) - United States Department of Agriculture (USDA). The document is available at: www.ers.usda.gov/publications/ldp/APR04/ldpm11801/. Accessed June 15, 2007. 
Haley, M. M. 2004. Market Integration in the North American Hog Industries. Electronic Outlook Report, ERS-USDA.

Hansen, B. E. 1999. “Testing for Linearity”. Journal of Economic Surveys 13: 551-576.

Horowitz, J. 2001. “The Bootstrap”. In J. J. Heckman and E. E. Leamer, eds. Handbook of Econometrics Vol. 5. Elsevier Science B.V.

Houck, J.P. 1977. "An Approach to Specifying and Estimating Non-reversible Functions". American Journal of Agricultural Economics 59: 570-572

Kwiatkowski, D., P. C. B. Phillips, P. Schmidt, and Y. Shin. 1992. Testing the Null Hypothesis of Stationarity Against the Alternative of a Unit Root: How Sure are we that Economic Time Series Have a Unit Root?”. Journal of Econometrics 54: 159-178.

Lass, D. A. 2005. "Asymmetric Response of Retail Milk Prices in the Northeast Revisited" Agribusiness 21: 495-508.

Luukkonen, R., P. Saikkonen, and T. Teräsvirta. 1988. "Testing Linearity against Smooth Transition Autoregressive Models". Biometrika 75: 491-499.

MacDonald, J. M., and M. E. Ollinger. 2000. "Scale Economies and Consolidation in Hog Slaughter". American Journal of Agricultural Economics 82: 334-346.

Maddala G. S., and I-M. Kim. 1998. Unit Roots, Cointegration and Structural Change. Cambridge: Cambridge University Press.

McCorriston, S., C. W. Morgan, and A. J. Rayner. 2001. "Price Transmission: The Interaction between Market Power and Returns to Scale". European Review of Agricultural Economics 28: 143-159.

Meyer, J., and S. von Cramon-Taubadel. 2004. "Asymmetric Price Transmission: A Survey". Journal of Agricultural Economics 55: 581-611.

Miller, D. J., and M. L. Hayenga. 2001. "Price Cycles and Asymmetric Price Transmission in the U.S. Pork Industry”. American Journal of Agricultural Economics 83: 551-562.

Park, J. Y., and P. C. B. Phillips. 2001. "Nonlinear Regressions with Integrated Time Series". Econometrica 69: 117-161.

Parker, C., E. Paparoditis, and D. N. Politis. 2006. "Unit Root Testing via the Stationary Bootstrap". Journal of Econometrics 133: 601-638.

Saikkonen, P. 1991. "Asymptotically Efficient Estimation of Cointegration Regressions". Econometric Theory 7: 1-21.

Saikkonen, P. 2005. "Stability Results for Nonlinear Error Correction Models". Journal of Econometrics 127: 69-81. 
Saikkonen, P., and I. Choi. 2004. "Cointegrating Smooth Transition Regressions". Econometric Theory 20: 301-340.

Seo, M. 2006. "Bootstrap Testing for the Null of No Cointegration in a Threshold Vector Error Correction Model”. Journal of Econometrics 134: 129-150.

Serra, T., and B. K. Goodwin. 2003. "Price Transmission and Asymmetric Adjustment in the Spanish Dairy Sector". Applied Economics 35: 1889-1899.

Shimotsu, K., and P. C. B. Phillips. 2005. "Exact Local Whittle Estimation of Fractional Integration. Annals of Statistics 33: 1890-1933.

Shimotsu, K. 2006. Exact Local Whittle Estimation of Fractional Integration with Unknown Mean and Time Trend. Working paper \#1061, Department of Economics, Queen's University.

Tsay, R. S. 1989. "Testing and Modeling Threshold Autoregressive Process". Journal of the American Statistical Association 84: 231-240.

Van Dijk, D., T. Tiräsvirta, and P. H. Frances. 2002. "Smooth Transition Autoregressive Models - A Survey of Recent Developments". Econometric Reviews 21: 1-47.

Wang, D., and W. G. Tomek. 2007. "Commodity Prices and Unit Root Tests". American Journal of Agricultural Economics 89: 873-889.

Ward, R.W. 1982. "Asymmetry in Retail, Wholesale and Shipping Point Pricing for Fresh Vegetables”. American Journal of Agricultural Economics 62: 205-212. 


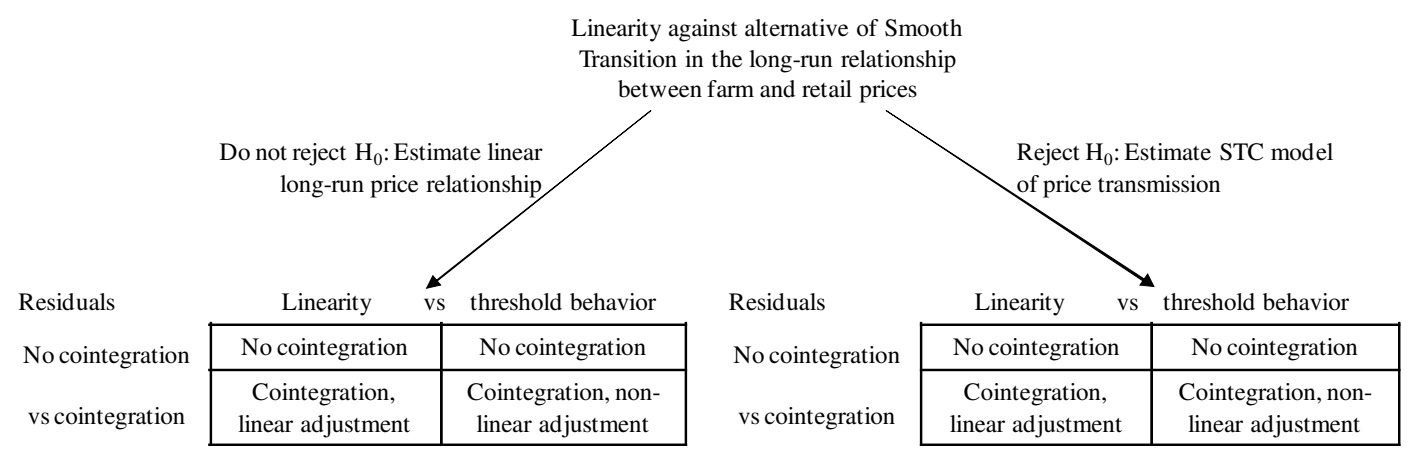

Figure 1. Steps to disentangle Non-linearities in the Long- and Short-run Price Relationships

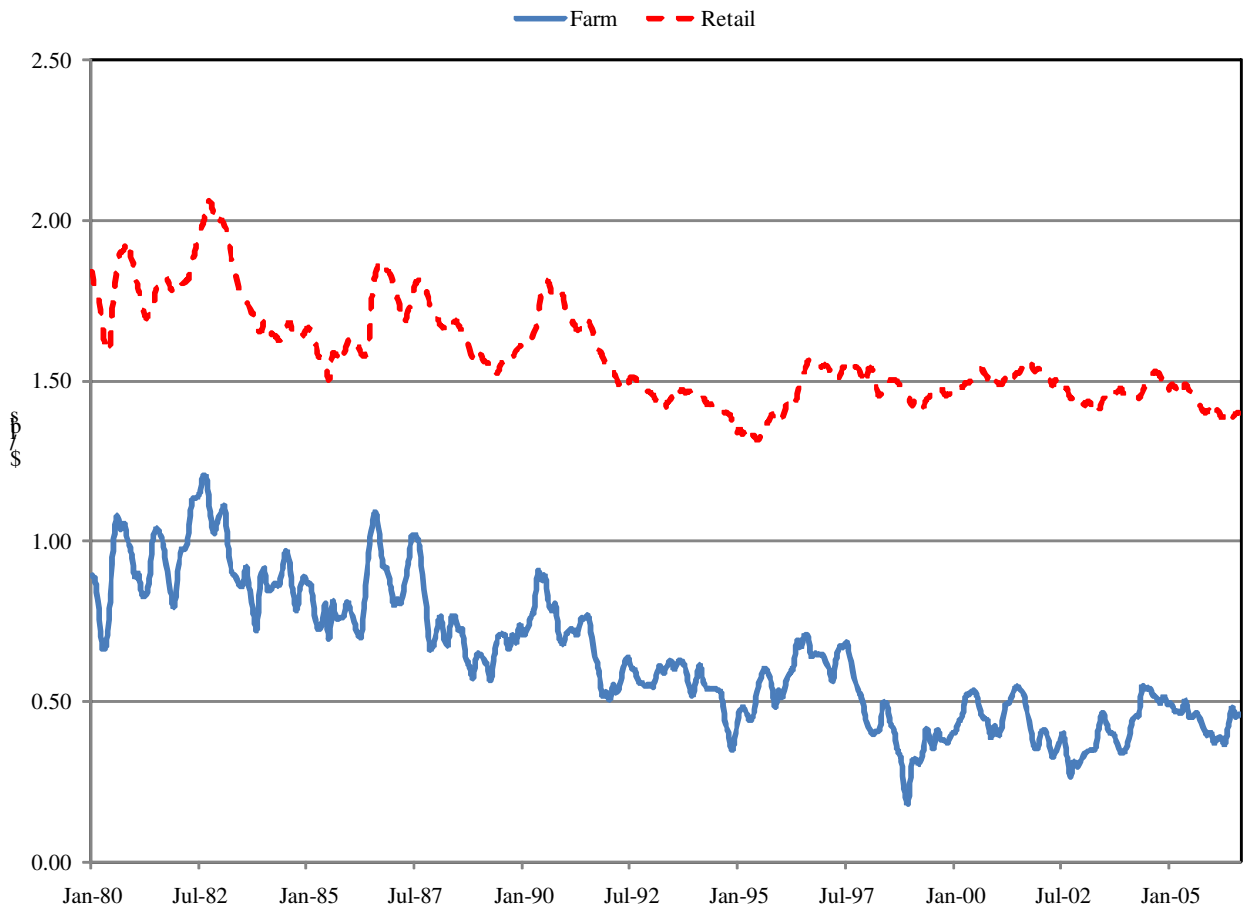

Figure 2. Monthly hog farm prices and pork retail prices in the U.S. in real terms, January 1980 - August 2006 


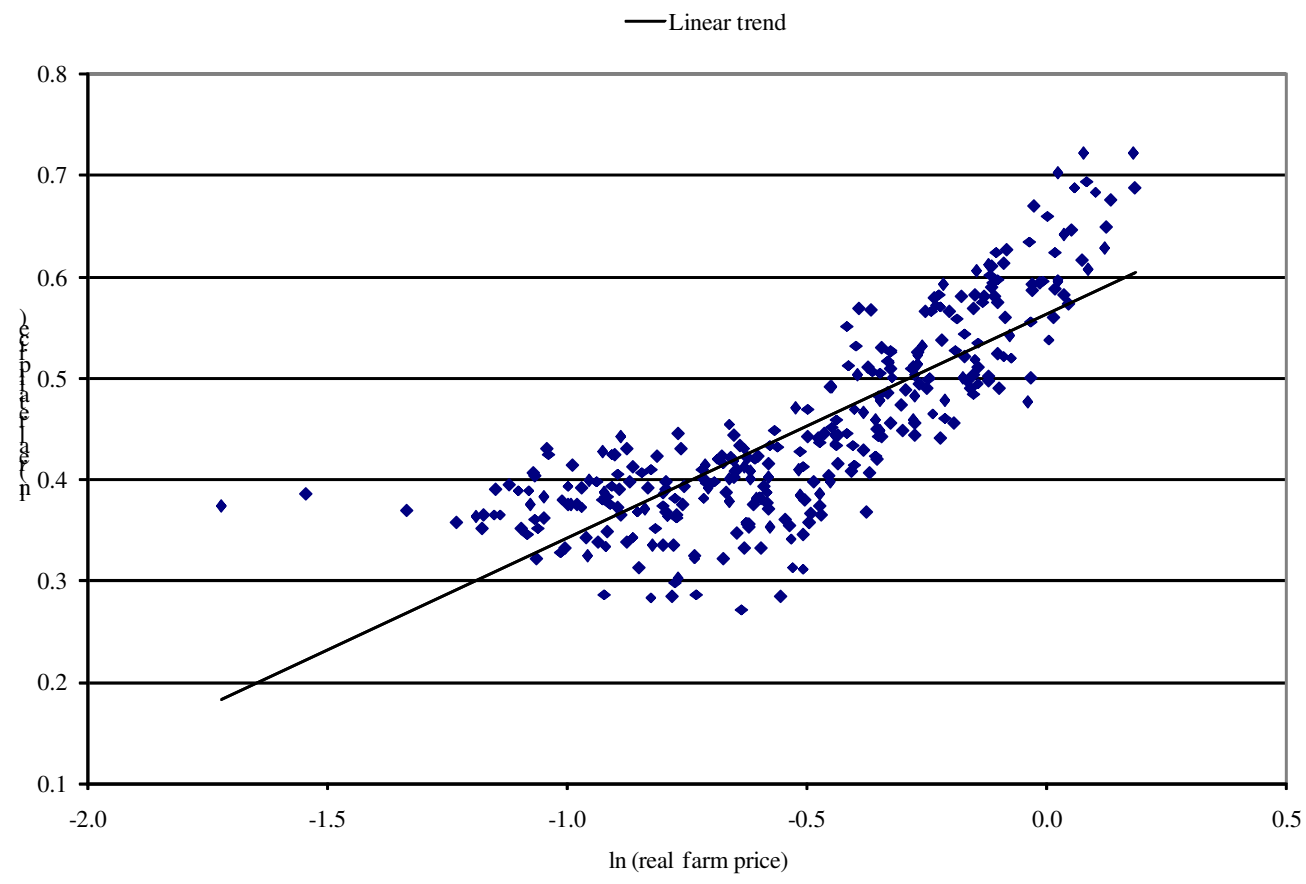

Figure 3. Farm and retail prices in the U.S. hog/pork sector, January 1980 - August 2006.

Table 1. Investigation of the Stochastic Properties of the Data

\begin{tabular}{|c|c|c|}
\hline Test statistic & Farm price & Retail price \\
\hline Bootstrap $p$-value of the null & & \\
\hline hypothesis of a unit root & 0.112 & 0.336 \\
\hline $\begin{array}{l}\text { Confidence interval for the } \\
\text { feasible ELW estimator of } d\end{array}$ & {$[0.909 ; 1.019]$} & {$[0.870 ; 0.980]$} \\
\hline $\begin{array}{l}\text { Confidence interval for the 2-step } \\
\text { feasible ELW estimator of } d\end{array}$ & {$[0.909 ; 1.019]$} & {$[0.870 ; 0.980]$} \\
\hline
\end{tabular}


Table 2. Non-Linearity Tests of the Cointegrating Vector

\begin{tabular}{|c|c|c|}
\hline Lag structure & $\tau_{1}$ statistic & $\tau_{2}$ statistic \\
\hline$K=1$ & 125.16 & 135.44 \\
\hline$K=2$ & 128.13 & 140.14 \\
\hline$K=3$ & 127.53 & 141.45 \\
\hline
\end{tabular}

Note: The $\tau_{1}$ and $\tau_{2}$ statistics follow a chi-squared distribution with respectively 1 and 2 degrees of freedom. The respective 5\% and $10 \%$ critical values are: $\chi(1)=3.84 ; \chi(2)=5.99$ and $\chi(1)=2.71$; $\chi(2)=4.61$.

Table 3. Estimates of the Smooth Transition Cointegration Model

\begin{tabular}{|c|c|c|}
\hline Parameters & Estimate & Standard error \\
\hline$\gamma$ & 5.92 & 1.34 \\
\hline$c$ & -0.69 & 0.04 \\
\hline$\alpha$ & 0.61 & 0.01 \\
\hline$\beta$ & 0.19 & 0.02 \\
\hline$\delta$ & 0.25 & 0.04 \\
\hline
\end{tabular}




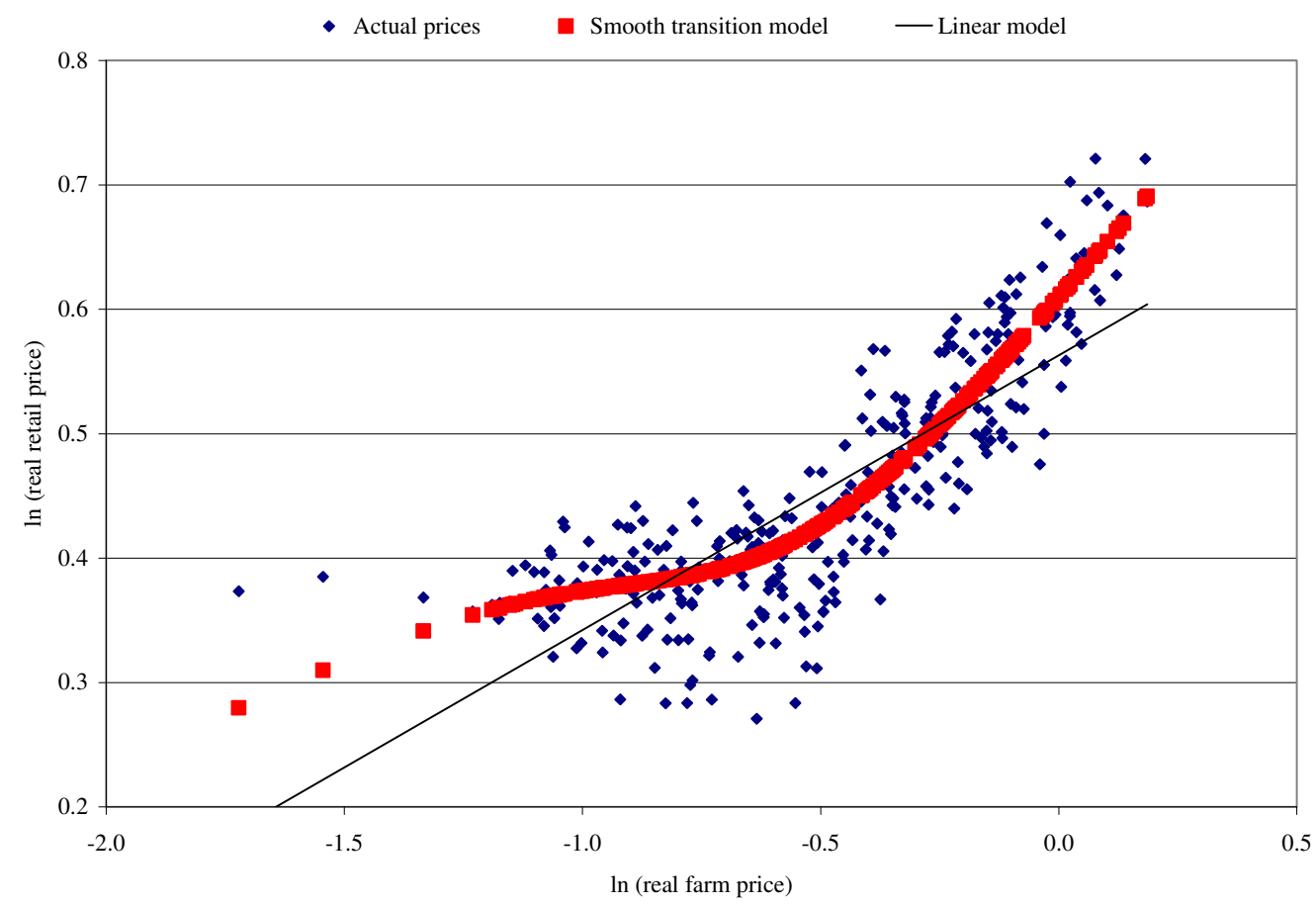

Figure 4. Linear and smooth transition cointegration models for the U.S. hog/pork supply chain

Table 4. Residual-Based Tests of Cointegration and Non-Linearity Using the Residuals from the Smooth Transition Cointegration Framework

\begin{tabular}{|c|c|c|c|}
\hline $\begin{array}{l}\text { Stationary } \\
\text { bootstrap } p \text { - } \\
\text { value }\end{array}$ & $\begin{array}{l}\text { Asymptotic } p \text { - } \\
\text { value of LST's } \\
\text { non-linearity test }\end{array}$ & $\begin{array}{l}\text { Asymptotic } p \text { - } \\
\text { value of Tsay's } \\
\text { linearity test }\end{array}$ & $\begin{array}{c}\text { Bootstrap p-value } \\
\text { of Hansen's } \\
F_{12} \text { test }\end{array}$ \\
\hline 0.000 & 0.153 & 0.537 & 0.146 \\
\hline
\end{tabular}

Table 5. Residual-Based Test of Cointegration and Non-Linearity Using the Residuals from the Linear Cointegration Framework

\begin{tabular}{|c|c|c|c|}
\hline $\begin{array}{l}\text { Stationary } \\
\text { bootstrap } p \text { - } \\
\quad \text { value }\end{array}$ & $\begin{array}{l}\text { Asymptotic } p \text { - } \\
\text { value of LST's } \\
\text { non-linearity test }\end{array}$ & $\begin{array}{l}\text { Asymptotic } p \text { - } \\
\text { value of Tsay's } \\
\text { non-linearity test }\end{array}$ & $\begin{array}{c}\text { Bootstrap p-value } \\
\text { of Hansen's } \\
F_{12} \text { test }\end{array}$ \\
\hline 0.000 & 0.001 & 0.456 & 0.466 \\
\hline
\end{tabular}


Table 6. Size of the Non-Linearity Tests Using the Residuals Generated by a Linear or Smooth Transition Cointegrating (STC) Vector when the True Data Generating Process is a STC Vector

\begin{tabular}{|c|c|c|c|c|c|c|}
\hline \multirow[t]{2}{*}{ Test } & \multicolumn{3}{|c|}{ Linear cointegrating vector } & \multicolumn{3}{|c|}{ STC cointegrating vector } \\
\hline & $1 \%$ & $5 \%$ & $10 \%$ & $1 \%$ & $5 \%$ & $10 \%$ \\
\hline Tsay & 0.108 & 0.276 & 0.394 & 0.001 & 0.006 & 0.015 \\
\hline Hansen & 0.124 & 0.272 & 0.383 & 0.001 & 0.004 & 0.013 \\
\hline LST & 0.612 & 0.792 & 0.858 & 0.062 & 0.229 & 0.376 \\
\hline
\end{tabular}

Table 7. Power of the non-linearity tests using the residuals generated by a linear or STC cointegrating vector when the true data generating process is a STC vector and the residuals follow a stationary TAR process

\begin{tabular}{|c|c|c|c|c|c|c|}
\hline \multirow[t]{2}{*}{ Test } & \multicolumn{3}{|c|}{ Linear cointegrating vector } & \multicolumn{3}{|c|}{ STC cointegrating vector } \\
\hline & $1 \%$ & $5 \%$ & $10 \%$ & $1 \%$ & $5 \%$ & $10 \%$ \\
\hline Tsay & 0.052 & 0.155 & 0.242 & 0.024 & 0.143 & 0.321 \\
\hline Hansen & 0.073 & 0.168 & 0.246 & 0.030 & 0.189 & 0.340 \\
\hline LST & 0.533 & 0.755 & 0.831 & 0.389 & 0.662 & 0.786 \\
\hline
\end{tabular}

